\title{
Subcutaneous drains reduce the surgical site infection after primary wound closure in stoma reversal patients
}

\begin{abstract}
Background: Surgical site infection (SSI) is one of the most common complications following stoma reversal with reported incidence of up to $41 \%$. Several techniques have been developed to reduce the risk of SSI, including delayed primary closure and purse-string suture (PSS). Subcutaneous drains have been reported to be effective for eliminating SSI in hepatobiliary and colorectal surgery. Non primary closure techniques require additional wound care, extra time and efforts than primary wound closure. Against this background stoma reversal cases were reviewed to determine the efficacy of subcutaneous drain in reducing the risk of SSI after primary closure of wound.
\end{abstract}

Methods: All patients who underwent stoma reversal were studied. All patients had stoma reversal with standard technique. All wounds were primarily closed however a drain was placed in subcutaneous space. Wounds were followed for any signs of infection. Drains were removed when drain output was serous and less than $10 \mathrm{ml}$. All patients were also interviewed telephonically 30 days after the surgery to inquire any signs of SSI.

Results: Seven patients underwent stoma reversal. Two patients had penrose drain while five patients had Jacksonpratt vacuum drain. Drains were removed on postoperative day 3-6 (mean 4.5). Sutures/staples were removed 8-15(mean 11.5). Mean duration of follow up was 27.4days (12-69 days). None of the patients (0/7) had SSI.

Conclusion: The study shows that subcutaneous drain is effective for reducing SSI in patients undergoing primary wound closure after stoma reversal. Patients don't require additional wound care as well which adds to their comfort and leads to better cosmetic results.

Keywords: stoma reversal, surgical site infection, subcutaneous drain, purse string suture, primary wound closure
Volume 6 Issue I - 2018

\author{
Jai P Singh \\ Department of Surgery, Oswego Hospital, USA
}

Correspondence: Jai P Singh, General Surgeon, Oswego Hospital, Assistant Clinical Professor, SUNY Upstate Medical University, I I OW 7th Street, Oswego, New York, USA, Tel 315342677I, Fax 3153422783, Email drjp04@gmail.com

Received: January 31, 2018 | Published: February 19, 2018
Abbreviations: SSI, surgical site infection; PSS, purse string suture; SVD, subcutaneous vacuum drain; POD, postoperative day

\section{Introduction}

Stoma reversal is a commonly performed procedure. Surgical site infection (SSI) is one of the most common complications following stoma reversal with reported incidence of up to $41 \%{ }^{1,2}$ Surgical site infection leads to increased medical cost, requires additional wound care and extra medications. It might also prolong length of hospital stay and adversely affect quality of life. ${ }^{3,4}$ Following an episode of infection there is increased risk of wound dehiscence, incisional hernia formation and poor wound healing. Surgical site infection would also increase the cost compared to unremarkable recovery. Because primary wound closure has a relatively high SSI rate, several techniques have been developed to reduce the risk of SSI, including delayed primary closure, ${ }^{2,7}$ and purse-string suture (PSS). ${ }^{3,8,9}$ Non primary closure techniques require additional wound care and occupy more time and effort than primary wound closure., Subcutaneous drains have been reported to be effective for eliminating
SSI, especially in hepatobiliary ${ }^{10}$ and colorectal surgery. ${ }^{11}$ Against this background stoma reversal cases were reviewed to determine the efficacy of subcutaneous drain in reducing the risk of SSI after primary closure of wound.

\section{Methods}

Patients who underwent stoma reversal at Oswego Hospital, New York were included in the study. All patients were operated by one surgeon.

\section{Preoperative care}

All stoma reversals were performed under general anesthesia. All patients were given weight based preoperative antibiotic dose within one hour of incision. Antibiotic dose was repeated during surgery if required according to the protocol. Patients who had reversal of colonic stoma were given both antibiotics and mechanical bowel preparation. Bowel preparation was not given to patients with ileostomy reversal. 


\section{Surgical technique}

The skin was prepared with povidone-iodine scrub and paint. An elliptical skin incision was given around the stoma and carried down to the subcutaneous tissue and then to the fascia. The stoma was separated from abdominal wall and resected. The anastomosis was done using staples. The wound was then closed in layers. Deep muscles were approximated using 2-0 absorbable sutures. Wound was irrigated with normal saline. Anterior fascia was then closed with either \#1 vicryl or \# 0 PDS sutures in transverse fashion. Wound was again irrigated with copious amount of normal saline. A drain (penrose or Jackson Pratt vacuum drain) was then placed over the fascia and fixed to the skin. All Drains were placed deep to the scarpa's fascia and superficial to the anterior rectus fascia. Penrose drain was brought out through the edge of incision while vacuum drain was brought out through a separate skin stab incision. Jackson Pratt drain was connected to the bulb for vacuum suction. The skin was then closed using skin staples or 2-0 non-absorbable sutures. Occlusive dressing was done with dry gauze and adhesive tape.

\section{Postoperative care}

Dressing was removed on POD \# 2 unless required to be changed prior to that due to saturation of dressing in order to keep incision site clean and dry. Vacuum drain bulb was emptied daily and output was measured, care was however taken to maintain vacuum suction. Drainage tube was milked everyday to remove any clot or occlusion. The drains were removed when the drainage was clear serous and less than $10 \mathrm{cc} / 24$ hours. All patients were seen by me every day until discharge from the hospital. All incisions were observed for any signs of SSI including redness, tenderness, in duration and purulent discharge. If the drain was not removed before discharge, then patients were discharged with drains in situ and were followed in the office. All sutures or staples were removed in the office along with drains if any. All patients were also interviewed telephonically 30 days after the stoma reversal to inquire any symptoms or signs of infection.

\section{Discussion}

Primary wound closure after stoma reversal has a relatively high rate of SSI and therefore several non-primary techniques have been studied to reduce the risk of wound infection, including leaving wound open, ${ }^{12}$ delayed primary closure ${ }^{2}$ and circumferential subcuticular wound approximation. ${ }^{9,13}$ Leaving the wound open and letting that healed by secondary intention significantly reduces the wound infectio, ${ }^{12}$ however these patients require prolonged wound care that adds to patients' discomfort and results in poor cosmesis. Delayed primary wound closure also requires wound care until the wound is closed, which again adds to patients' discomfort and requires second procedure. Another technique, subcuticular PSS has been widely used and encouraging outcomes have been reported. ${ }^{1,9,13-15}$ In a prospective randomized controlled trial, Duschet al included 84 patients with ileostomy reversal and compared direct sutures with PSSs. They found that the rate of SSI was significantly lower after PSS $(0 \%)$ compared with direct suture $(24 \%) .{ }^{14}$ Another randomized study by Reid $\mathrm{K}$ et al showed that purse-string closure resulted in significantly fewer SSIs than conventional direct closure (2/30 vs. $12 / 31 ; p=0.005) .{ }^{15}$ In PSS technique, size of the wound is decreased by circumferential wound approximation however an opening is still left in the center for effusion to drain. The open wound would still require additional wound care until the wound is completely healed by secondary intention leaving poor cosmetic scar. Effective drainage is critical for preventing surgical site infection. Facilitating drainage and preventing formation of subcutaneous dead space are the fundamental mechanisms of all non-primary skin closure techniques. After the primary closure, patients require less wound care and the healing is faster compared to delayed closure. ${ }^{3}$ In the present study the incision was primarily closed over the drain rather than leaving it open. The incidence of SSI was $0 \%$ in our group, which is comparable with that of PSS. Theoretically, subcutaneous drains are effective in extracting effusion and eliminating the dead space of the wound following stoma reversal. Several studies have reported that subcutaneous drain is effective for reducing SSI. ${ }^{10,11}$ Fujiietal reported thatthe incidence of SSI in patients undergoing colorectal surgery was $14.3 \%$ in subcutaneous drain group vs $38.6 \%$ in no drain group. ${ }^{11}$ Baier PK et al. ${ }^{16}$ however did not find a relationship between the use of subcutaneous drain and decreased incidence of SSI after laparotomy. ${ }^{16}$ Primary wound closure after stoma reversal has an even higher risk of SSI as surgical field is more likely to get contaminated compared to other abdominal surgeries. Pan HD et al studied the role of subcutaneous vacuum drain after primary wound closure in patients with ileostomy reversal. They found that the patients with drain had lower SSI rate vs the patients with no drain $(1.2 \%$ vs $12.5 \%) .^{3}$ In the current study although the sample size was small however the wound infection rate was $0 \%$. Subcutaneous drains were very effective in preventing SSI in all seven patients following a procedure that carries high risk of SSI. Results support that primary closure with subcutaneous drain is an alternative choice other than purse string wound closure for stoma reversal. In my practice I have switched over to subcutaneous vacuum drains from Penrose drains as the use of SVD also decreased the need of dressing change postoperatively because the effusion was continuously extracted through the suction drain rather than through the surface of the wound. Future prospective randomized trials are required to compare subcutaneous drains with PSSs in stoma reversal patients (Figures 1-4).

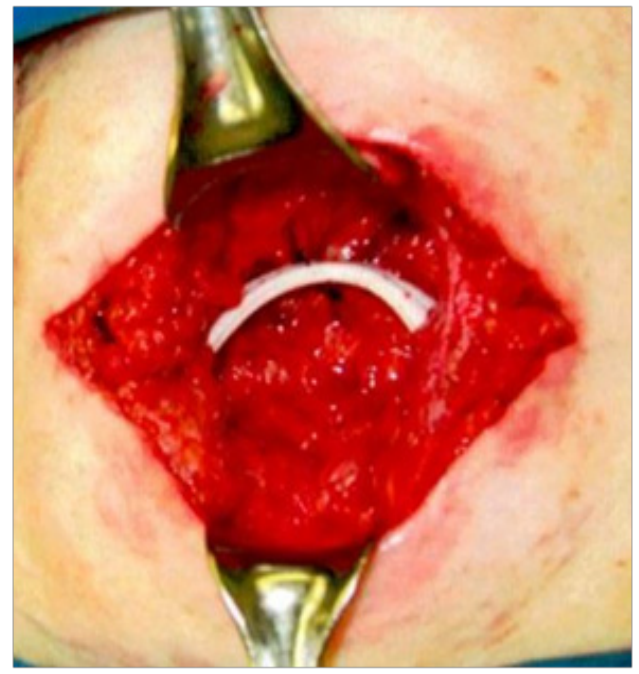

Figure I Subcutaneous vacuum drain over the closed fascia (patient 4). 


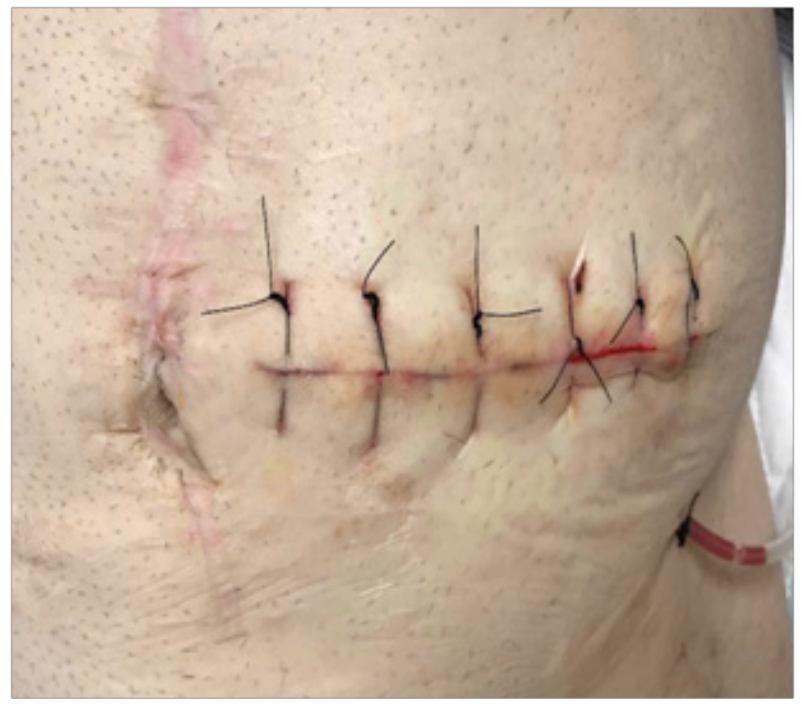

Figure 2 Incision on POD\#5 with no evidence of SSI (patient 4).

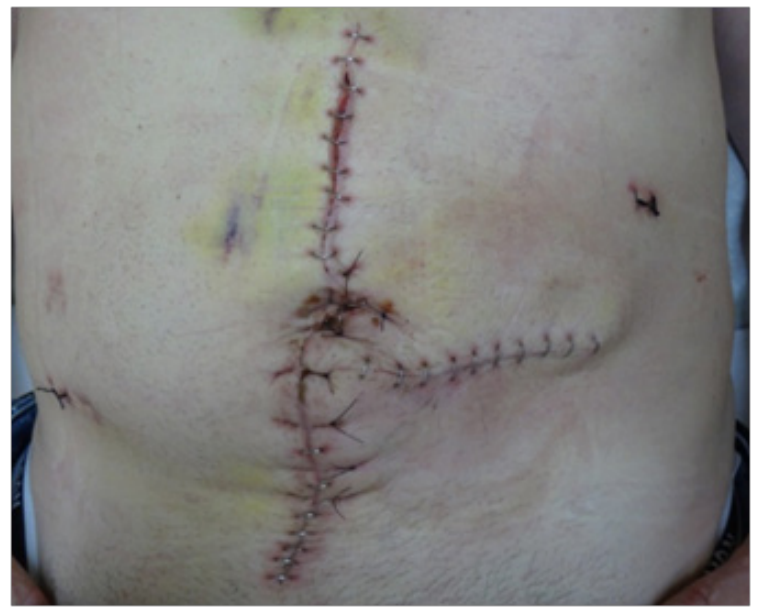

Figure 3 Incision on POD\#I0, no signs of SSI (patient 5).

Table I Patient characteristics

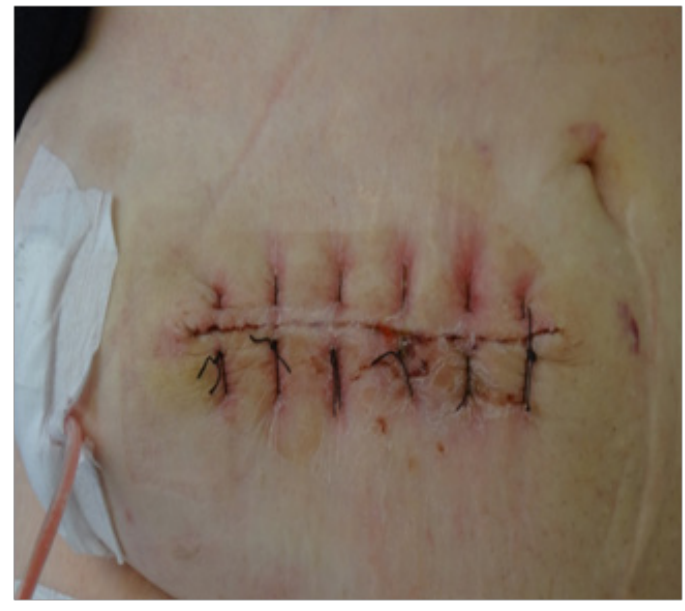

Figure 4 Incision on POD\#5, no signs of SSI (patient 7).

\section{Results}

Total seven patients $(n=7)$ underwent stoma reversal. Six patients had reversal of loop ileostomy and one patient had reversal of end colostomy. The patient who had reversal of end colostomy had initial surgery as sigmoid colectomy with end colostomy for sigmoid perforation. Before his colostomy closure he had colonoscopy as preoperative work up and was found to have a large cecal polyp that could not be removed endoscopically. Therefore at the time of colostomy reversal he had right hemicolectomy with primary ileotransverse anastomosis along with colostomy take down and colorectal anastomosis. Mean age was 56years (50-66years). The mean interval between creation and closure of ostomy was 134.8days (68-238days). The mean BMI was 31.8(25.4-45.9). Two patients had Penrose drain and five patients had Jackson pratt drain. Drains were removed on postoperative day \# 3-6 (mean 4.5). Six patients had their drains removed before their discharge from the hospital and remaining one patient had his drain removed on first follow up visit in the office. Sutures/staples were removed on postoperative day 8-15 (mean 11.7). Mean duration of follow up was 27.4days (12-69days), however all patients were telephonically followed after 30days. None of the patients had SSI (0/7) (Table 1$)$.

\begin{tabular}{|c|c|c|c|c|c|c|c|c|c|}
\hline Patient & Age & Sex & BMI & Diabetes & Smoking & Drain type & $\begin{array}{l}\text { Drain removed } \\
\text { (POD) }\end{array}$ & $\begin{array}{l}\text { Sutures/staples } \\
\text { removed (POD) }\end{array}$ & SSI \\
\hline 1 & 53 & $\mathrm{~m}$ & 29,5 & no & yes & Penrose & 6 & 13 & No \\
\hline 2 & 66 & $\mathrm{f}$ & 25.4 & no & no & Penrose & 4 & 13 & No \\
\hline 3 & 53 & $\mathrm{~m}$ & 27,5 & no & no & jp & 4 & 9 & No \\
\hline 4 & 50 & $\mathrm{~m}$ & 26.9 & yes & no & jp & 5 & 10 & No \\
\hline 5 & 59 & $\mathrm{~m}$ & 29.3 & no & no & jp & 5 & 14 & No \\
\hline 6 & 55 & $\mathrm{~m}$ & 27,2 & no & yes & jp & 3 & 8 & No \\
\hline 7 & 62 & $\mathrm{f}$ & 45.9 & yes & no & jp & 5 & 15 & No \\
\hline Mean & 56 & & 31.8 & & & & 4.5 & 11.7 & \\
\hline
\end{tabular}




\section{Conclusion}

In conclusion, this study shows that subcutaneous drain is effective for reducing SSI in patients undergoing primary wound closure after stoma reversal. Additionally patients don't require wound care as well which avoids the discomfort and leads to better cosmetic results.

\section{Acknowledgements}

None.

\section{Conflict of interest}

The author declares no conflict of interest.

\section{References}

1. Li LT, Hicks SC, Davila JA, et al. Circular closure is associated with the lowest rate of surgical site infection following stoma reversal: a systematic review and multiple treatment meta-analysis. Colorectal Dis. 2014;16(6):406-416.

2. Lahat G, Tulchinsky H, Goldman G, et al. Wound infection after ileostomy closure: a prospective randomized study comparing primary vs. delayed primary closure techniques. Tech Coloproctol. 2005;9(3):206-208.

3. Pan HD, Wang L, Peng Y, et al. Subcutaneous vacuum drains reduce surgical site infection after primary closure of defunctioning ileostomy. Int J Colorectal Dis. 2015;30(7):977-982.

4. Taylor GD, Kirkland TA, Mckenzie MM, et al. The effect of surgical wound infection on postoperative hospital stay. Can J Surg. 1995;38(2):149-153.

5. Murray BW, Cipher DJ, Pham T, et al. The impact of surgical site infection on the development of incisional hernia and small bowel obstruction in colorectal surgery. Am J Surg. 2011;202(5):558-560.

6. Mahmoud NN, Turpin RS, Yang G, et al. Impact of surgical site infections on length of stay and costs in selected colorectal procedures. Surg Infect (Larchmt). 2009;10(6):539-544.
7. Hackam DJ, Rotstein OD. Stoma closure and wound infection: an evaluation of risk factors. Can J Surg. 1995;38(2):144-148.

8. Banerjee A. Pursestring skin closure after stoma reversal. Dis Colon Rectum. 1997;40(8):993-994.

9. Milanchi S, Nasseri Y, Kidner T, et al. Wound infection after ileostomy closure can be eliminated by circumferential subcuticular wound approximation. Dis Colon Rectum. 2000;52(3):469-474.

10. Tsujita E, Yamashita Y, Takeishi K, et al. Subcuticular absorbable suture with subcutaneous drainage system prevents incisional SSI after hepatectomy for hepatocellular carcinoma. World J Surg. 2012;36(7):1651-1656.

11. Fujii T, Tabe Y, Yajima R, et al. Effects of subcutaneous drain for the prevention of incisional SSI in high-risk patients undergoing colorectal surgery. Int J Color Dis. 2011;26(9):1151-1155.

12. Vermulst N, Vermeulen J, Hazebroek EJ, Et al. Primary closure of the skin after stoma closure. Management of wound infections is easy without (long-term) complications. Dig Surg. 2006;23(4):255-258.

13. Lee JR, Kim YW, Sung JJ, et al. Conventional linear versus purse-string skin closure after loop ileostomy reversal: comparison of wound infection rates and operative outcomes. J Korean Soc Coloproctol. 2011;27(2):5863.

14. Dusch N1, Goranova D, Herrle F, et al. Randomized controlled trial: comparison of two surgical techniques for closing the wound following ileostomy closure: purse string vs direct suture. Color Dis. 2013;15(8):1033-1040

15. Reid K1, Pockney P, Pollitt T, et al. Randomized clinical trial of short-term outcomes following purse-string versus conventional closure of ileostomy wounds. Br J Surg. 2010;97(10):1511-1517.

16. Baier PK1, Glück NC, Baumgartner U, et al. Subcutaneous Redon drains do not reduce the incidence of surgical site infections after laparotomy. A randomized controlled trial on 200patients. Int $J$ Colorectal Dis. 2010;25(5):639-643 